\title{
HACIA UN MODELO PARA LA GENERACIÓN AUTOMÁTICA DE INTERFACES GRÁFICAS CENTRADAS EN EL USUARIO
}

\author{
Pedro Omar Silva-Vásquez ${ }^{1}$, Edgard Benítez-Guerrero ${ }^{2}$ y Viviana Yarel Rosales-Morales ${ }^{3}$ \\ ${ }^{1}$ Universidad Veracruzana - CONACYT \\ ${ }^{2}$ Universidad Veracruzana \\ ${ }^{3}$ Cátedras CONACYT - Universidad Veracruzana \\ Facultad de Estadística e Informática, Av. Xalapa s/n Obrero Campesina 91020 Xalapa-Enríquez, Ver. México
}

\begin{abstract}
RESUMEN
En los últimos años se han presentado trabajos en ingeniería de software para el desarrollo de aplicaciones de diversas índoles, sobre todo en dos áreas principales: el modelado de la aplicación y la generación automática de código. Aunque estas aplicaciones generadas automáticamente pueden ser de gran utilidad para agilizar los tiempos de desarrollo, se ha dejado de lado la apariencia de la interfaz gráfica de usuario (GUI por sus siglas en inglés) y la interacción, la cual no necesariamente cumple con los requerimientos de interacción esperados por los usuarios finales porque solo se toma en cuenta la funcionalidad de la aplicación. Por otra parte, el diseño centrado en el usuario (DCU), es un enfoque al diseño y desarrollo de sistemas interactivos donde se busca hacerlos más usables. Tomando en cuenta los conceptos del DCU, en este documento se propone un nuevo modelo que plantea como objetivo principal, además de la generación automática de interfaces gráficas de usuario, que se contemple el diseño centrado en el usuario durante la generación automática de las mismas. Para esto se analizaron los trabajos relacionados con la generación automática de software y de código, el DCU y la usabilidad.
\end{abstract}

\section{PALABRAS CLAVE}

Diseño Centrado en el Usuario, Interfaz Gráfica de Usuario, Generación Automática de Software, Usabilidad, Interacción, Experiencia del Usuario

\section{INTRODUCCIÓN}

En el área de la Ingeniería de Software se han presentado trabajos para el desarrollo de aplicaciones de diversas índoles, los cuales buscan un desarrollo de software eficaz y eficiente. Esto ha conllevado a que se desarrollen aplicaciones utilizadas para generar la aplicación y la generación automática de código; sin embargo, estos desarrollos solo toman en cuenta la funcionalidad de la aplicación, pero esto no asegura que la interfaz gráfica de usuario cumpla con los conceptos de un diseño centrado en el usuario (DCU).

En los documentos consultados para esta investigación, particularmente Marín, Giachetti y Pastor, (2007), Rosales-Morales et al., (2019) y Panach et al. (2007), presentan propuestas de generación automática de código para aplicaciones. De estos, en ninguno se toma en cuenta el diseño centrado en el usuario, enfocándose en la funcionalidad de la aplicación y proponiendo maneras en las que se puede agilizar el tiempo de desarrollo. Sin embargo, es importante considerar el DCU para la interacción del usuario final, porque una GUI centrada en el usuario ayuda a determinar si la aplicación es útil, usable y posteriormente deseable (Nielsen, 2012).

Durante la investigación no se ha encontrado una sola técnica o método de recolección de datos para los requisitos que hagan que una aplicación tenga un diseño centrado en el usuario en la primera entrega al usuario final, y las metodologías utilizadas suelen ser muy lentas, ya que conllevan diversos pasos: primero se realiza un análisis de los requerimientos del usuario para conocer sus necesidades, después se presenta la propuesta de diseño, y esto se complica si el análisis de requerimientos fue ejecutado de manera incorrecta, lo cual conllevaría a tener que realizar todo el proyecto de nuevo para poder agregar las correcciones del usuario final. 
El ciclo del diseño centrado en el usuario se puede apreciar en la figura 1. El primer paso, marcado de color verde, es la identificación de las necesidades del usuario. El segundo paso es comprender y especificar el contexto del uso. El tercer paso es especificar el usuario y los requerimientos organizacionales, mientras que el cuarto paso es el diseño de la solución, mismo que en el quinto paso se evalúa contra los requerimientos solicitados por el usuario. Finalmente de ser esto correcto, en el sexto paso se termina ya que el sistema satisface los requerimientos específicos del usuario y su organización. Si no se cumplen con los requerimientos específicos del usuario y de la organización, se debe comenzar de nuevo el ciclo.

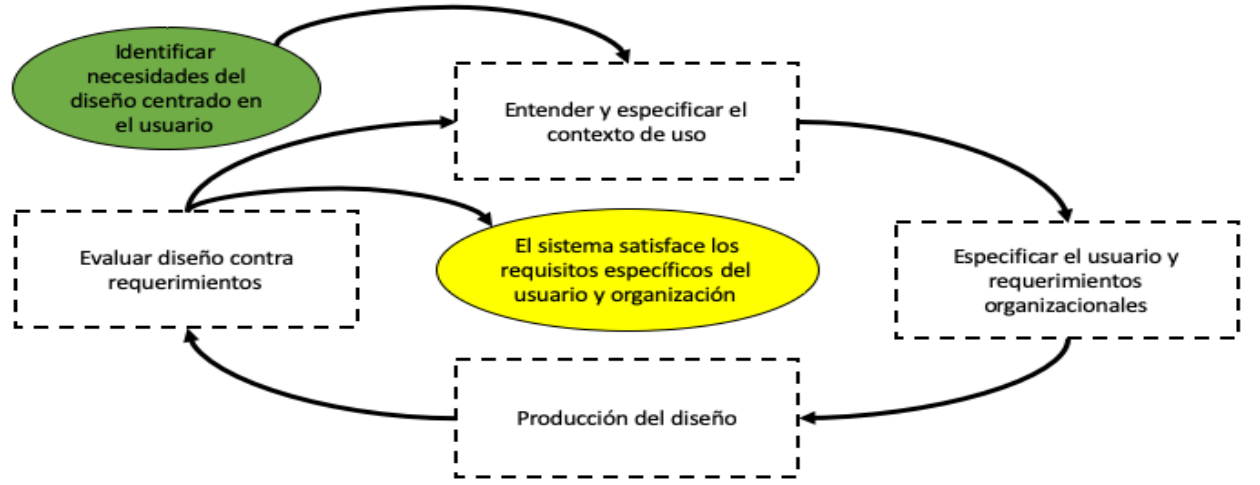

Figura 1. Ciclo del diseño centrado en el usuario. Fuente: Sánchez, (2011)

Esta metodología para el diseño centrado en el usuario sirve para evidenciar que en los trabajos relacionados que se centran en la generación automática de código no se ha considerado la importancia del DCU, ya que las aplicaciones antes mencionadas se centran en agilizar el proceso de desarrollo de sistemas y aplicaciones, pero no consideran la usabilidad y apariencia, incumpliendo con los requerimientos de diseño que espera el usuario. Una vez que se cumplen con las funcionalidades de la aplicación, si el sistema muestra un mal diseño de distribución, mal manejo de colores, saturación de mensajes, o si el usuario debe dar más clics de los necesarios, terminará por ser tediosa para el usuario final y caer en desuso.

Como se puede apreciar el proceso del DCU no es lineal, y para ser evaluado Nielsen (2000) considera que las evaluaciones con 5 usuarios solo se podrían alcanzar un 80\% de los problemas y con 15 participantes, se puede alcanzar el $100 \%$ de los problemas de usabilidad, estas pruebas suelen ser muy lentas y costosas, perdiendo el objetivo primordial que es acortar el tiempo en el que se realiza el diseño.

El resto de este documento presenta un modelo que se someterá a pruebas y evaluación, el documento está organizado de la siguiente manera, la sección 2 presenta los trabajos relacionados a los temas de interés. La sección 3 describe el modelo propuesto para solucionar el problema planteado de la generación automática de aplicaciones. Por último, la sección 4 concluye este documento.

\section{ANTECEDENTES}

El objetivo de los antecedentes es fundamentar la falta de implementación del DCU en trabajos de generación automática de código. En el aspecto de desarrollo automático de interfaces se encuentra la propuesta de Rosales-Morales et al., (2019) que presenta a ImagIngDev, que es un enfoque para el desarrollo de aplicaciones móviles multiplataforma que utiliza técnicas de procesamiento de imágenes, que se basa en reconocer patrones de diseño de interfaz de usuario para generar el código. En esta propuesta encontraron beneficios de desarrollo ya que permite a los usuarios generar aplicaciones móviles multiplataforma y multi-dispositivo de manera fácil e intuitiva. Para ello presentan las fases del proceso automático de desarrollo de las aplicaciones móviles, donde definen ciertos patrones que debe seguir el usuario que realiza el trazado de los dibujos para no generar discrepancia entre lo que entra y lo que debe generar una vez que ha sido identificado. Estos elementos pasan a la fase de configuración en la que se selecciona la plataforma y configuraciones de la misma, esta fase culmina en la generación de un archivo XML que mandará a la siguiente fase que es la generación de código basado en lo establecido en el XML y la presentación del mismo. No se contemplan los requerimientos de DCU para el usuario final. 
La propuesta que expone Marín, Giachetti y Pastor, (2007), a pesar de su antigüedad se le considera de importancia ya que llevaron a cabo un arduo trabajo más extenso que integra la generación automática de código y la incorporación del DCU. En este trabajo presentan la relación entre modelos UML y "OO-method". Para esto ellos proponen una arquitectura en UML para poder unificar con el OO-method. Una vez que se tiene la conversión se transforma en un archivo XML para que se pueda convertir en XMI (XML Metadata Interchange). Una vez listo el archivo, se ingresa en OLIVANOVA Modeler que es una herramienta de edición y validación de modelos conceptuales OO-method. En la propuesta de Molina y Pastor, (2005) se integra el XMI para que se obtenga la aplicación final, la cual no considera en ninguno de sus puntos de desarrollo las necesidades del usuario y sus interacciones.

En la búsqueda de trabajos relacionados con la usabilidad y la generación automática de códigos se puede mencionar a Panach et al. (2007) con OO-sketch, que es una propuesta que toma como base el trabajo de Marín, Giachetti y Pastor, (2007), los autores de OO-sketch ocupan los modelos de tarea para generar un diagrama de interacción y poder entender las actividades que debe realizar el usuario, buscando que cumpla con los criterios de usabilidad en el proyecto que se va a generar, estos diagramas deberán apoyar el contexto de función y aplicación que debe ejecutarse en el software diseñado. Aunque ellos generan estos diagramas, no mencionan si evaluaron estos diagramas en un proyecto con usuario finales dando la aprobación y avalando el DCU en la aplicación final.

Cabe mencionar que durante el tiempo de la investigación no se han reportado trabajos que además de generar las aplicaciones o las interfaces gráficas de usuario contemplen el diseño centrado en el mismo y satisfagan todas las necesidades de diseño, por lo que el modelo que se propone considera aspectos que insertan el diseño centrado en el usuario en el proceso de la generación automática de código.

\section{MODELO PARA LA GENERACIÓN AUTOMÁTICA DE INTERFACES GRÁFICAS CENTRADAS EN EL USUARIO}

Considerando los aspectos mencionados anteriormente sobre la falta de aplicación del DCU en la generación automática de software, se propone un nuevo modelo (presentado en la figura 2). Este modelo representa la generación automática de interfaces gráficas centradas en el usuario, el cual inicia con el "usuario diseñador" de la aplicación que puede ser el analista, el ingeniero en software o el programador, para este caso nosotros lo llamaremos "diseñador". Después de esto en la primera fase se encuentra el análisis de requerimientos, consta de los requisitos funcionales y los requisitos no funcionales, para brindarles una presentación formal.

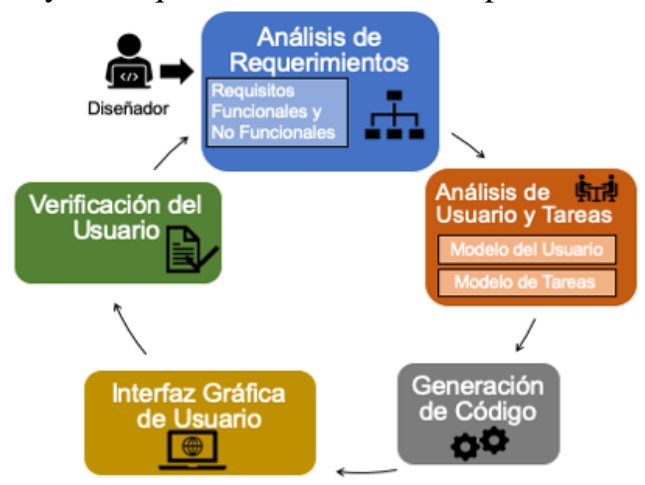

Figura 2. Modelo para la generación automatica de interfaces gráficas centradas en el usuario

En la siguiente fase, que es la del análisis del usuario y tareas, se integra el modelo del usuario que para Hassan (2004) es toda la información obtenida de usuarios, para definir la clase o perfiles basándonos en atributos comunes, estos harán la clasificación dependiendo de la información de la audiencia, pero se tratarán atributos como necesidades de información, condiciones de acceso, experiencia y conocimientos éste se agrega por que es parte importante del DCU. Se tiene también el modelo de tareas del usuario, representado en un Concur Task Tree (o por sus siglas CTT) que funcionará para modelar las tareas del usuario en una aplicación interactiva. Este tipo de modelo ayuda a realizar una aplicación intuitiva, su sintaxis gráfica la hace sencilla de interpretar. En esta podemos encontrar 4 tipos de tareas en función del 
actor que las llevará a cabo: tareas de usuario, tareas de aplicación, tareas de interacción y las tareas abstractas, así es como lo define Zumaquero, (2009), se considera integrarlo por los antecedentes donde se ha utilizado este modelo.

Una vez que se tengan estas propiedades analizadas se visualiza en una formalización de la aplicación en conjunto para evaluarla por el usuario. Ya que están de acuerdo ambas partes se pasa a la fase de ejecución para la generación automática de código, para así obtener una aplicación generada automáticamente con un DCU, una vez generada la aplicación la GUI se le muestra al usuario final para que él determine si le parece correcta su presentación, la verifique y la apruebe en caso de no hacerlo se regresa a la parte de análisis de requerimiento reiniciando el ciclo.

\section{CONCLUSIONES Y TRABAJOS FUTUROS}

Los trabajos reportados anteriormente se han centrado en agilizar el trabajo del desarrollo de aplicaciones, con lo cual es posible agilizar el desarrollo de éstas, pero ignoran una parte importante, que es la interfaz gráfica del usuario, siendo esto lo primero que visualiza el usuario y debería cobrar mayor importancia. Debido a esto, aunque la aplicación cumpla con todos los requerimientos de función solicitados por el usuario, si la interacción no tiene un diseño apropiado (centrado en el usuario), ésta se convierte en un aspecto mal calificado por el usuario final. Por esta razón el modelo presentado en este documento es de suma importancia para la generación automática de código y de interfaces gráficas centradas al usuario.

Como trabajo futuro se tiene contemplado realizar una evaluación del modelo propuesto con un caso de estudio, se está trabajando en el área de aplicaciones educativas centradas en el usuario para validar si lo propuesto contribuye en la agilización para obtener aplicaciones que cumplan con los criterios de usabilidad.

\section{AGRADECIMIENTO}

Este trabajo fue apoyado parcialmente por el Consejo Nacional de Ciencia y Tecnología (CONACYT) de México en el marco del proyecto de Cátedras "Infraestructura para Agilizar el Desarrollo de Sistemas Centrados en el Usuario" (Ref. 3053). Agradecemos también a CONACYT por la beca de Doctorado número 395377 del primer autor, así como a la Universidad Veracruzana por el apoyo para el desarrollo de esta investigación.

\section{REFERENCIAS}

Marín, B., Giachetti, G. and Pastor, O. (2007) 'Intercambio de modelos UML y OO-method', MEMORIAS: 10th Workshop Iberoamericano de Ingenieria de Requisitos y Ambientes de Software - IDEAS 2007, (May 2014), pp. 283-296.

Molina, J. and Pastor, O. (2005) 'MDA, OO-Method y la Tecnología OLIVANOVA Model Execution', (May).

Nielsen, J., 2000. Why You Only Need to Test with 5 Users. [En línea] disponible: https://www.nngroup.com/articles/why-you-only-need-to-test-with-5-users/

Nielsen, J., 2012. Usability 101: Introduction to Usability. [En línea] disponible: https://www.nngroup.com/articles/usability-101-introduction-to-usability/

Panach, J. I. et al. (2007) 'OO-sketch: Una herramienta para la captura de requisitos de interacción', MEMORIAS: 10th Workshop Iberoamericano de Ingenieria de Requisitos y Ambientes de Software-IDEAS 2007, (May 2014), pp.357-370.

Rosales-Morales, V. Y. et al. (2019) 'ImagIngDev: A New Approach for Developing Automatic Cross-Platform Mobile Applications Using Image Processing Techniques', The Computer Journal. doi:10.1093/comjnl/bxz029.

Sánchez, J., 2011. En busca del Diseño Centrado en el Usuario(DCU): definiciones, técnicas y una propuesta. No Solo Usabilidad. [En línea] http://www.nosolousabilidad.com/articulos/dcu.htm?utm_source=iNeZha.com\&utm_medium=im_robot\&utm_campa ign=iNezha

Zumaquero, S. F., 2009. Aplicación de la notación CTT (ConcurTaskTrees) a la creación de rutas en un museo, Albacete, España: SYMBIA IT. 